\title{
Tecnologías de información en el diseño de la organización
}

\author{
Andrade, Jesús Alberto* \\ Campo-Redondo, María Susana**
}

\section{Resumen}

Las tecnologías de información forman parte importante de las organizaciones modernas. La influencia reciproca de la organización y las tecnologías de información, se ve reflejada cuando se estudia la automatización y el grado de participación que las tecnologías tienen para resolver problemas de productividad. En particular, los mecanismos de reestructuración y reingeniería para alcanzar el diseño organizacional, son los enfoques más utilizados para lograr mejoras en el funcionamiento de los sistemas de información. Este artículo analiza las ventajas y desventajas que tiene en el diseño organizacional el uso de tales enfoques. Además, se desarrolla la idea de que el diseño de los sistemas de información debe estar centrado en lo humano.

Palabras clave: Reingeniería, reestructuración, sistemas de información, tecnologías de información, productividad, ventajas competitivas, empleo.

Recibido: 98-09-08 : Aceptado: 98-10-27

* Economista, La Universidad del Zulia. MSc. Computación Aplicada en La Universidad del Zulia, LUZ. MSc. Gerencia de Sistemas de Información Computarizada en Eastern Michigan University EMU. Profesor Titular del Programa de Gerencia y Gestión Tecnológica en la Facultad Experimental de Ciencias FEC en LUZ. Miembro del Departamento de Matemáticas y Computación de la FEC, LUZ. Maracaibo, Venezuela. Email: jandrade luz.ve

** Psicólogo, Universidad Rafael Urdaneta. MSc. en Orientación en La Universidad del Zulia, LUZ. MSc. Psicología Clínica en Eastern Michigan University EMU. Profesora Asociada del Programa de Posgrado en Orientación en LUZ. Miembro del Departamento de Psicología de la Facultad de Humanidades y de Educación de LUZ. Maracaibo, Venezuela Email marisucampo @yahoo.com 


\section{Informational Technologies in Organizational Design}

\section{Abstract}

Informational technology forms an important part of modern organizations. The reciprocal relationship between organizations and informational technology is reflected in the study of automatization and the level of participation which the technologies employ in the solution of productivity problems. In particular, the mechanisms of restructuring and reengineering in order to affect organizational design, are the most common focuses used to improve the functioning of information systems. This article analyzes the advantages and disadvantages that the use of these focuses has in organizational design. Furthermore, the idea that the design of information systems should be centered on the human element, is also developed.

Key words: Reengineering, restructuring, information systems, information technology, productivity, competitive advantages.

\section{Introducción}

Desde la década de los sesenta, la aparición masiva de tecnologías de información - TI- ha ocupado un lugar predominante en el espectro organizacional. Desde entonces, el uso de computadores en tareas consideradas esenciales para el proceso productivo ha delineado el horizonte del diseño de la organización, caracterizado por una permanente innovación en las TI y en la forma como las organizaciones realizan las tareas para alcanzar sus metas y objetivos. En particular, las TI han sido vistas como herramientas esenciales para alcanzar mejoras en la productividad. Bajo ese enfoque utilitarista, se ha pretendido incrementar la productividad mejorando los procesos a través de la automatización. Como consecuencia de esto, una explosión informacional se ha dado en el entorno de la organización, poniéndole presión a los sistemas de información -SI-, generando crisis en la productividad de los mismos, y extendiendo la creencia, por parte de la gerencia, que existen soluciones mágicas a problemas de orden técnico y organizativo.

Este trabajo analiza el impacto de las TI en el diseño de la organización, con especial énfasis en la utilización de reestructuración y reingeniería ya que estos son dos de los enfoques que se utilizan con mayor frecuencia en aquellas organizaciones que tratan de mejorar la productividad de sus sistemas de información. En tal sentido, se profundiza en el análisis de los conceptos de reestructuración y de reingeniería, debido a que existe una diversidad de interpretaciones que en ocasiones conducen a utilizar en forma errónea tales términos. Se analiza además, la 
necesidad de que las organizaciones se planteen el diseño de los sistemas de información dentro de la estrategia de la organización. Por ultimo, se desarrolla la idea a modo de conclusión, de que los sistemas de información automatizados deben ser desarrollados considerando el factor humano.

\section{La organización y su influencia en las aplicaciones de tecnologías de información}

Una gerencia orientada hacia la creación, adquisición, implantación, difusión y mantenimiento de la tecnología informática ayuda a alcanzar las metas y los objetivos de la organización. Ello se logra, primero fortaleciendo la importancia de la innovación junto a la colaboración tecnológica que la gerencia esté dispuesta a gestionar, y segundo, permitiendo el alcance de ventajas competitivas.

Uno de los principales factores que determina el desempeño de las organizaciones modernas está medido por el grado de automatización que la empresa ha desarrollado en sus sistemas de información. La relación existente entre organización y tecnología ha sido estudiada desde mucho tiempo atrás y la mayoría ha concluido que la tecnología influye intensamente en el desempeño organizacional. Wilkinson (1983) estudió la relación existente entre tecnología y organización, y señaló cómo la tecnología puede ser moldeada por el contexto social. En particular, afirma que la manera de escoger una determinada tecnología es un proceso político, ya que las máquinas pueden envolver una particular forma de ejercer control en la organización.
Para algunas tecnologías y en particular para las $\mathrm{TI}$, la fase del diseño tecnológico solamente ocurre despues que ha sido identificada una aplicación específica, en un determinado contexto social, (Le Quesne,1988:395). Y es que el ambiente organizacional puede tener un efecto significante en la forma como se expresa una determinada tecnología de información, algo que no es posible con otros tipos de tecnología. Así pues, la forma final como se manifiesta la tecnología de información dependerá de la naturaleza del ambiente organizacional.

Itami (1987) considera que las organizaciones cuentan con dos tipos de recursos o activos, los visibles tangibles, y los invisibles intangibles. Los activos visibles como el hardware de las organizaciones son fundamentales para el funcionamiento de la organización, pero resultan estériles si no se cuenta con un activo invisible como el software de las aplicaciones. Los activos visibles tangibles se reproducen a través de inversiones de capital o flujos de dinero, tales como las máquinas y equipos que manejan data. En particular, los activos intangibles crecen gracias a los flujos de información que se generan en la organización y no en función de otros recursos tangibles como el dinero. Cuando los miembros de una organización son capaces de procesar, absorber, y digerir información, a través del aprendizaje de errores y éxitos propios o de sus competidores, están generando flujos de intormación que la empresa reproduce en su organización.

En las TI relacionadas con el desarrollo de software, una aplicación se define como una manifestación dentro del contexto de la organización, expresada 
en un flujo de información. La selección de las tareas y la creación de los programas son responsabilidad de los individuos cercanos a la organización donde se instalará la nueva TI, por ende el resultado final de la aplicación de esa tecnología es una expresión final de su organización. Por lo tanto, el ambiente de la organización tiene un efecto significativo en la forma final como se manifiesta la TI.

\section{Grado de automatización}

El grado de automatización es comúnmente usado para medir qué tan eficientemente una organización está usando sus recursos. Existe una tendencia a pensar que todo proceso debe ser automatizado, a fin de lograr una respuesta de los sistemas de información que sea mejor y más rápida. Existen sobradas razones para suponer que la automatización de los procesos conllevará a una mejora sustancial de la organización; al fin de cuentas, se pretende mejorar la forma como se realizan las cosas en ella. Hacer las cosas mejor tiene como objetivo principal mejorar la eficiencia de los procesos, lo que significará para la organización una oportunidad de reconfigurar el trabajo a fin de servir mejor a sus clientes, y como consecuencia obtener mejoras y beneficios para todos aquelios interesados en la organización, tales como los accionistas, o cualquier otro ente que participe internamente de la organización.

Según Ayres (1991:352), las empresas pretenden aumentar su productividad automatizando labores manuales con el único fin de producir veinticuatro horas al día sin parar. Esto se hace con éxito en aquellas tareas repetitivas y sis- temáticas que ocurren en las fábricas, ya que busca reflejarse en una disminución en los costos de mano de obra.

En las oficinas, donde se desarrollan trabajos administrativos, se pretende aplicar el mismo concepto que se ha aplicado en la fábrica: automatizar procesos, también con la esperanza de alcanzar ahorros en los costos del recurso humano que se dedica a labores manuales del procesamiento de datos o en tareas que requieren tediosos cálculos; sin embargo, tales ahorros se ven superados por los costos de automatización, en la reposición periódica de maquinaria, la contratación de personal más especializado, y en el mantenimiento de los componentes de la TI, entre los que destaca el software de aplicaciones.

Recientemente, siguiendo la línea marcada por los productos informáticos para las oficinas, las organizaciones tienden a comprar productos informáticos con aplicaciones que son el núcleo de las operaciones estándares a utilizar en el trabajo rutinario, productos que brindan funcionabilidad de acuerdo a las especificaciones que les exige el sector donde la organización se desenvuelve. Aplicaciones de compra, inventario, o sistemas completos relacionados con las operaciones bancarias o de seguros, forman parte de la gama de productos disponibles, muchas veces mejor diseñados que los anteriores sistemas monolíticos que se desarrollaban internamente. Una ventaja de que esto ocurra, es que garantiza que se están aplicando los mejores procesos y las mejores prácticas profesionales de trabajar en el área, a la vez que se utiliza el software del mercado, de entendimiento masivo. El recurso humano especiali- 
zado en informática se encarga entonces, de instalar el software a la medida de las necesidades de la organización. Esta práctica revela como las organizaciones se adaptan a las líneas del mercado y en especial a los vaivenes de las $\mathrm{TI}$, en lugar de ser las tecnologías de información las que se adapten a las necesidades de la organización. Ello representa, en algunos casos, un aspecto negativo para mejorar la productividad, ya que los individuos dentro de la organización se sienten disconformes al permitir que el software marque el rumbo y dicte la pauta de cómo se debe comportar la organización.

\section{Mejora en el funcionamiento de los sistemas de información}

La automatización en las oficinas no es buena ni mala per se, es en todo caso, una manera de realizar las labores de la organización. Sus bondades están enmarcadas en la búsqueda por aumentar la calidad de los productos de información, bien sea disminuyendo el tiempo de respuesta, o eliminando los errores de data. En este sentido, en cuanto a los sistemas de información se refiere, una revisión de sus funcionamientos debe focalizar en dos aspectos primordiales: en mejorar sus tiempos de respuesta para hacerlos más expeditos, y en una constante búsqueda por la eliminación de pasos que burocratizan los procesos. Para ello, las empresas enfocan el diseño organizacional básicamente desde dos ángulos:

a) aplicación de reestructuración, y

b) aplicación de reingeniería.
Para una mejor comprensión de los términos mencionados anteriormente dentro del contexto de este trabajo, se requiere definirlos en relación a las organizaciones que aplican ingeniería de software y desarrollan sistemas de información automatizados. La literatura en este aspecto es muy diversa y amplia. Existe una variedad de definiciones que muchas veces se contradicen, tales definiciones de reingeniería incluyen, según Feiler (1993):

- El examen y la alteración de un sistema existente, para reconstituirlo en una nueva forma y la subsecuente implantación de una nueva.

- El proceso de adaptar un sistema existente a cambios en su ambiente o tecnología sin cambiar su funcionabilidad general.

- Modificación y posible desarrollo posterior de un sistema existente.

- Mejora de un sistema a través de la aplicación de un reverso de ingeniería (y restructuración) seguida por una ingeniería de avance (hacla adeIante).

Hammer y Champy establecen ciertos principios que marcan el norte de sus ideas, y aunque algunas de ellas pudieran considerarse ya propuestas por los proponentes de la Gerencia de Calidad Total (CT), su principal crédito está centrado en el hecho de que ellos focalizan en los resultados y no en la organización, a la vez que desestiman la importancia de la atención que podría dársele a la gente que se dedica a labores de nivel operacional bajo, o aquellos que están ubicados por debajo de la alta gerencia. En este sentido, reingenjería rechaza la necesidad del entrenamiento continuo de 
quienes están en las tareas del día a día, ya que la organización no debe funcionar con aquella noción de Adam Smith basada en tareas simples que se deban asignar a especialistas, sobre la división del trabajo, las economías de escala y el control jerárquico que según Hammer y Champy es lo que hacen los administradores de las organizaciones de hoy, al focalizar sus actividades en tareas, oficios, personas y estructuras, pero no en procesos. Desde un punto de vista estrictamente gerencial, afirman que las ideas que ellos desarrollan son tan importantes para las organizaciones de hoy, como aquellas de los últimos dos siglos desarrolladas por Adam Smith en relación a la empresa y la gerencia.

Es interesante destacar lo que Hammer y Champy (1994:50) observan sobre lo que no es la reingeniería. Establecen claramente que no se debe confundir la reingenieria de negocios con la llamada reingeniería de software, que significa (para ellos) reconstruir sistemas obsoletos de información con tecnología más moderna. Al margen de las consideraciones que estos autores hagan de lo que no es reingeniería es importante destacar que su teoría se basa en la mejora (radical) de los procesos, que son apropiados para la aplicación de métodos sistemáticos de trabajo. En esencia existe un reconocimiento a la importancia que tienen los procesos que se desarrollan en la organización. Aseguran que para poder aplicar informática se debe pensar en forma inductiva, primero reconocer una solución poderosa, y luego buscar problemas donde aplicarla.

Contrario a la noción de Hammer y Champy acerca de lo que es la reingenie- ría de software, los especialistas en desarrollo de software son mucho más específicos en cuanto a su significado, ya que desarrollan el concepto de reingeniería y reestructuración como una técnica y no como parte de una concepción teórica. Pressman (1992) define el concepto de reingeniería a partir de ingeniería de reversa el cual tiene su origen en el mundo del hardware. Históricamente se refiere al proceso aplicado en el desarme de ciertos componentes de hardware en un esfuerzo por entender los secretos de diseño y manufactura de los competidores. Ingeniería de reverso para software es bastante similar, aunque la mayoría de las veces los programas no son de los competidores, sino que pertenecen a la organización que los usa. De preferencia se aplica a programas y procesos que no fueron desarrollados con metodologías documentales, o programas y procesos que fueron desarrollados mucho tiempo atrás que carecen de documentación. Se intenta recuperar el diseño del programa, la data y los procedimientos, involucrando a los requerimientos funcionales del sistema, es decir, las necesidades actuales de los usuarios del mismo. El esfuerzo se concentra en analizar un programa y tratar de crear una representación del programa en un nivel más alto que el escrito en lenguaje de código de máquina, de forma tal que sea entendible por los seres humanos.

Para Pressman (1992:681) reingeniería o renovación, o reclamación (Chikofsky, 1990 citado por Pressman), pretende no solamente recuperar el diseño y la información asociada al software existente, tal como se logra con ingeniería de reversa, sino que además, tal informa- 
ción sirve para alterar o reconstituir el sistema existente en un esfuerzo por mejorar la calidad de todo el producto. En la mayoría de los casos, reingeniería de software reimplementa las funciones de los sistemas existentes. Pero al mismo tiempo, quien desarrolla software, añade nuevas funciones y mejora el rendimiento de los ya existentes.

El Instituto de Ingeniería de Software (SEI -siglas en inglés-) de la Universidad de Carnegie Mellon, que asiste al Departamento de Defensa de los Estados Unidos en la aplicación de reingenieria a sistemas que usan software en forma intensiva, considera necesario utilizar una taxonomía que defina los términos para alcanzar una evolución exitosa de la tecnología de software. En tal sentido el SEI desarrolló tal taxonomía ${ }^{1}$ que actualmente es de aceptación común.

El Gráfico 1 ilustra algunos términos relacionados con reingeniería definidos por Chikofsky. Según Feiler (1993:3-5) tales abstracciones son representadas en términos de las fases del ciclo de vida de un sistema de información. El proceso tradicional de desarrollar un sistema es conocido como ingeniería de avance (forward engineering). Ingeniería de reversa (reverse engineering) es el proceso de analizar un sistema existente; identificando los componentes del siste$\mathrm{ma}$, las abstracciones y las relaciones. Reestructuración (restructuring) es la transformación de representaciones al mismo nivel de abstracción mientras se preserva la conducta externa del sistema. Reingeniería (reengineering) es un proceso de ingeniería para reconstituir un sistema existente en una nueva forma a través de una combinación de ingeniería de reversa, reestructuración e ingeniería de avance. En tal sentido reingeniería está asociado a la fase de mantenimiento del sistema de información, que está generalmente visto como conformado por corrección, perfeccionamiento, prevención, y mantenimiento adaptativo.

Como el gráfico lo indica, la reestructuración puede darse en forma aislada en las distintas fases que se imponen en el cambio organizacional o como parte de un proceso de reingeniería de la organización.

En atención a la conceptualización anteriormente desarrollada, existe una contradicción entre lo propuesto por Pressman, Chikofsky, y SEI, y lo propuesto por Hammer y Champy en el sentido de lo extenso y el momento en que se debe aplicar reingeniería. Ya que para Pressman, Chikofsky, y SEl el momento ideal para aplicar reingeniería, ocurre

Taxonomía elaborada a partir del trabajo de Chikofsky, de los resultados del primer Workshop del Grupo de Políticas de Coordinación en Gerencia de Recursos de Computación, con sede en Santa Bárbara California, y de otros institutos y centros de investigación internacional como son la Agencia de Proyectos de Investigación Avanzada (Advanced Research Projects Agency -ARPA-) en Estados Unidos y EUREKA (Eureka Software Factory), así como del Instituto para Tecnologias de Software y Sistemas (Institute for Systems and Software Technology) de Frauenhofer Gesellschaft con sede en Europa. 
Grático 1

Algunos Términos Relacionados con Reingeniería

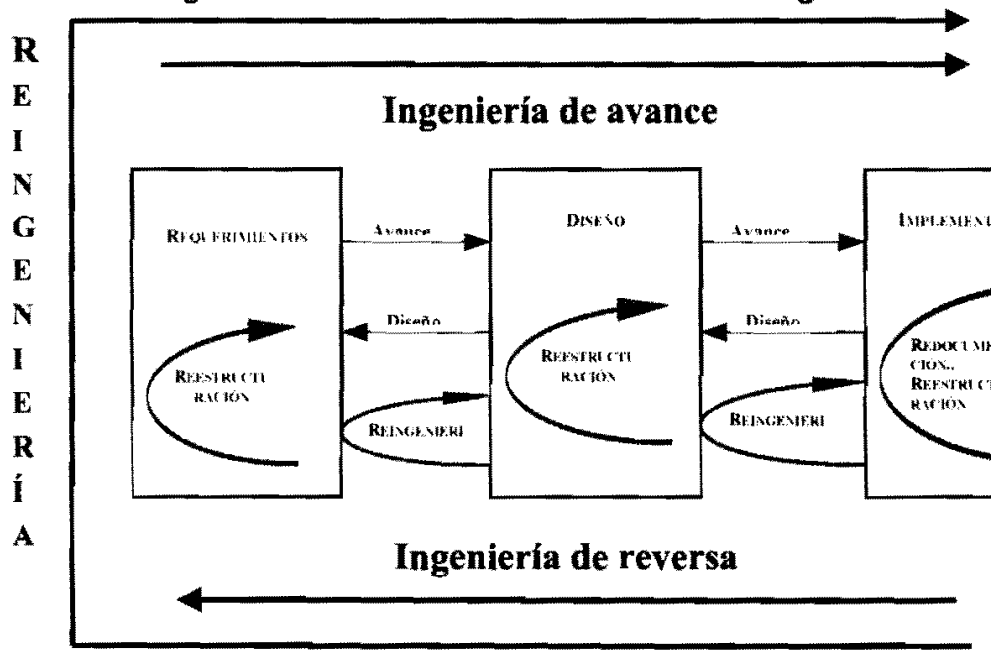

Tomado de Feiler (1993) Special Report CMU/SEI-93-SR-5 Reengineering y adaptado por los autores.

cuando se requiere hacer tareas de mantenimiento de software lo cual implícitamente indica que se refiere a programas que están en ejecución y que requieren una mejora en el diseño del proceso, o la incorporación de un nuevo enfoque a una tarea que se ha venido ejecutando rutinariamente. Pressman (1992:681) señala tres aspectos importantes que se deben considerar al momento de aplicar reingeniería:

1. en organizaciones que tienen millones de líneas de código de software elaborado y que son candidatos a la aplicación de reingeniería, sería irreal realizarlo por diversos motivos, señalando entre otros, que la frecuencia de uso de tales programas puede no ameritar el cambio;

2. las herramientas disponibles para aplicar reingeniería al software son para un determinado tipo de aplicaciones, y no para todo tipo; $y$
3. el costo y el esfuerzo podría hacer prohibitiva su aplicación.

Para Hammer y Champy (1994), reingeniería es la revisión fundamental y el rediseño radical de procesos para alcanzar mejoras espectaculares en medidas críticas de rendimiento. Afirman que la reingeniería no puede ser ejecutada en pequeños pasos y en forma continua. Es una propuesta de todo o nada que produce resultados dramáticos. Se olvida por completo de lo que es y se concentra en lo que debe ser. Llegar hasta la raíz de las cosas: no efectuar cambios superficiales ni tratar de arreglar lo que ya está instalado sino abandonar lo viejo. Se deben dar saltos gigantescos en rendimientos ya que no se trata de hacer mejoras marginales. Se debe apelar a la reingeniería únicamente cuando exista la necesidad de volar todo. Proponen un cambio radical, fundamental y espectacular en todos 
los procesos, al menos en aquellos procesos que requieran una explosión pesada. Estos autores se preguntan por qué diseñamos procedimientos tan ineficientes. Para ellos, muchos de los procedimientos (en el estilo de gerencia norteamericana) no fueron diseñados, simplemente ocurrieron (Hammer, 1990:110).

Este trabajo considerará la propuesta taxonómica de Chikofsky desarrollada por la SEI y la IEEE COMPUTER SOCIETY, ya que unifica términos en el campo del software y de los sistemas de información de las organizaciones que desarrollan procesos automatizados, convirtiéndose en el estándar de hecho para la aplicación de ingeniería de software. Se evaluará el impacto que produce la aplicación de tales conceptos en lo que comunmente se entiende como reingenieria en las organizaciones, tal como lo proponen Hammer y Champy, pero focalizados en aquellas organizaciones que utilizan en forma intensiva procesos automatizados. De tal forma que no se intenta desarrollar ideas relacionadas con la reingeniería de procesos en su forma genérica, sino en el contexto específico de procesos automatizados. Se trata de un análisis profundo de aquellas organizaciones cuyos sistemas automatizados están funcionando; pero que requieren de mejoras importantes, así como también se analiza la repercusión que esta tiene en la organización una vez aplicado un diseño organizacional.

\section{Diseño organizacional a través de la aplicación de reestructuración}

Si un sistema de información puede ser reducido en su número de pasos, obviamente se conseguirá una mejora en la velocidad del tiempo de respuesta del sistema, por lo que la naturaleza de la reestructuración está enfocada en el número de pasos a reducir, dirigido a controlar las variables de la organización. Su efectividad se mide en término de cálculos de radios tales como número de módulos que mantener, número de módulos por sistema, número de llamadas a determinados procesos, y otros relacionados con estimaciones de resultados, lo que permite establecer o medir la reducción de costos.

Las empresas que aplican reestructuración a sus sistemas de información focalizan más su atención en la disminución del número de módulos que se despliegan en la estructura del sistema. Haciendo una revisión a través del enfoque Bottom-up (estrategia informal de diseño que permite concatenar diversos módulos en uno solo de mayor grado de abstracción), se revisan procesos que ocurren en un determinado SI. Procesos que forman parte del sistema se van concatenando con la idea de integrarlos en un único módulo. Tareas que son realizadas por un variado número de personas o procedimientos, pueden integrarse en un solo proceso. Así, mientras menos procesos se tengan que realizar, mayor será la reducción de los costos de los SI. La idea es preservar la conducta externa del sistema, aunque se realicen transformaciones internas de los procesos que conforman un mismo nivel de abstracción.

La técnica de reestructuración focaliza en los procesos organizativos y en su distribución en la estructura de la organización, por lo tanto, la reestructuración manipula los procesos que se desarrollan en las distintas unidades que están representadas en la organización. Reestructu- 
rar significa reconfigurar los procesos de las unidades de la organización, convirtiendo varios procesos (tanto manuales como automatizados) en un solo bloque de funciones. Si esto ocurre, la reestructuración puede conllevar a una modificación del trabajo, aún a costa del desplazamiento de mano de obra laboral a otras actividades operativas, $o$ en otros casos extremos a una pérdida de posiciones laborales. Se intenta así incrementar la eficiencia de los $\mathrm{Sl}$, a través de cambios significativos en el número de procesos que se realizan en la estructura organizacional.

En ocasiones, la reestructuración puede significar reducir el número de capas jerárquicas, a medida que se fusionan antiguos procesos en otros más amplios y generalizados, las tareas que eran desarrolladas por un particular número de capas burocráticas son ahora realizadas por un número menor de personas, lo que podría significar una sobre carga de actividades y responsabilidades del nuevo proceso, en menos manos. $Y$ aunque esto podría significar una mejora en el bienestar de los accionistas de la organización (debido a la disminución de los costos de personal), no necesariamente significará una mejora en los procesos, o un bienestar en la atención a los clientes, ya que estos podrían sentirse, al principio, mejor atendidos al existir menos intermediarios; sin embargo, al sobrecargarse de actividades los empleados que se quedan en la organización, la relación y la eficiencia laboral tenderá a deteriorarse. La naturaleza de la reestructuración es numérica (disminución de módulos) que busca aumentar la eficiencia focalizando en el control organizacional. Debido a ese interés por mantener con- trol sobre los distintos niveles jerárquicos se producen traumas en la organización.

El énfasis de la reestructuración se hace en beneficio de la organización, sin tratar de que esos beneficios tengan necesariamente que extenderse a los clientes. Su visión es a corto plazo y conlleva a la necesaria disminución del personal, lo cual es relativamente fácil ya que se trata de un recurso humano dedicado a labores de soporte de los flujos de información que pueden ser aprendidos con relativa facilidad por quienes se quedan.

\section{Diseño organizacional a través de la aplicación de reingeniería}

Reingeniería de los SI es el proceso de analizar un sistema internamente, en un esfuerzo por crear una representación del programa de aplicación a un nivel más alto de abstracción con el propósito de hacerlo más eficiente. Reingeniería representa una oportunidad para desarrollar a mediano plazo un plan de evolución del sistema de información en cuestión, además de sentar las bases para la evolución de familias de sistemas, que en lo futuro podrían ser desarrollados.

Desde un punto de vista gerencial, reingeniería es el análisis del sistema actual, considerando sus metas, y los factores que en lo futuro permitirán medirlo. Ya hemos señalado que para Hammer y Champy (1994) desde el punto de vista organizacional reingeniería significa un cambio radical (no efectuar cambios superficiales, ni tratar de arreglar lo que ya está instalado) del proceso organizativo ya que su fin es alcanzar mejoras dramáticas en la ejecución de las actividades que se realizan en la organización, de forma tal que refleje un beneficio en los cos- 
tos, en la calidad del servicio, y en la velocidad del desempeño laboral de las actividades administrativas.

¿Cómo repercute esa visión gerencial en el desarrollo de los sistemas de información y desarrollo de software en las organizaciones?. Existe una clara tendencia, por parte de quienes desarrollan aplicaciones de software, en tratar de mejorar procedimientos existentes a fin de aumentar la eficiencia en sus aplicaciones. La mayoría de las veces, el análisis y posterior aplicación de reingeniería, ocurre en unidades o departamentos que son muy vulnerables y que están pasando por situaciones que tienden al caos. Si la organización ha estado en actividad por mucho tiempo, seguramente ha ido desarrollando aplicaciones y procedimientos sofisticados que se van sobreponiendo como capas dentro de la organización, logrando impactos no deseados en las metas y objetivos organizacionales. Debe entonces hacerse un análisis profundo de los procesos automatizados. En tal sentido, hace falta desarrollar reingeniería a los procesos en una forma drástica. Si ese caso ocurre a nivel de los procesos automatizados, la reingeniería de software no solamente revisa los procesos para mejorarlos, sino que aplica nuevas formas que enriquecen la aplicación de tales procesos. Tal como lo señala Pressman (1998:506), existe una relación cíclica entre las capacidades de las tecnologías de la información (TI) y la reingeniería de procesos que se produce dentro de un negocio. A medida que crecen las capacidades de $\mathrm{Tl}$, pueden generar cambios en los procesos de negocios. Afirma que la reingeniería de procesos de negocios es iterativa, los objetivos de nego- cios, y los procesos que los logran, deben adaptarse a un entorno cambiante.

En la práctica, las empresas se concentran en aplicar programas de reingeniería a procesos, con el fin de mejorar la forma como se desarrollan las cosas internamente en la organización. Este análisis y diseño de los procedimientos parte de sistemas de información ya existentes, con un marcado énfasis en la aplicación de la técnica del Top Down (estrategia informal que utiliza el enfoque incremental, paso a paso, para analizar módu los de un sistema) para detallar cuáles procesos requieren de mejoras en su funcionamiento.

La realización de mejoras en los procesos significa innovación en la forma como funciona en la actualidad el proceso, por lo que la innovación es una de las mejores herramientas de que dispone la organización para redescubrir mejoras sustanciales que se reflejen en un aumento de la eficiencia de los procesos. Al aplicar reingeniería a las organizaciones se busca hacer el trabajo más fácil y más rápido, con menos pasos y con menor cantidad de recurso humano, así que otra de las consecuencias que se logra con la aplicación de reingeniería es la reducción de personal, particularmente de aquel que está en los niveles más bajos. La naturaleza de este tipo de diseño está orientado a lograr control sobre los aspectos técnicos y mantener autonomía de las variables de la organización, lo que en ocasiones produce ansiedad entre el personal.

Este nuevo diseño surgido luego de un proceso de reingeniería de software, y trasladado a la organización significa una solución que mejora la calidad de las actividades, al fin y al cabo si el ob- 
jetivo es hacer mejor las cosas, es de suponer que los procesos mejoran en término de la velocidad con que se realizan, y en la cantidad de recursos empleados, ya que su punto de enfoque se centra en las funciones de la organización y en la forma como se realiza el trabajo. Reingeniería para diseñar la organización focaliza en la aplicación de las mejores técnicas que se puedan aplicar a los procesos, a fin de beneficiar tanto a los integrantes de la organización como a los clientes, ya que busca la eficiencia interna y la satisfacción de los usuarios del sistema.

\section{Ventajas y desventajas de reestructuración y reingeniería en el diseño organizacional}

Ya hemos señalado la importancia de desarrollar reingeniería de software a procesos automatizados que así lo requieran, sin embargo, la noción de mejorar el proceso automatizado implica mejoras graduales, y lo que se pretende desde el punto de vista organizacional al aplicar reingeniería es alcanzar un cambio radical. Desde la perspectiva de este trabajo nos queda preguntarnos, ¿cómo se mide lo explosivo que pudiera ser un cambio radical de un proceso automatizado para una organización?. Lo cierto es que cambios drásticos, pudieran generar caos drásticos. Muchos de los cambios drásticos que requieren las organizaciones que hacen uso intensivo de software en sus sistemas de información, son consecuencia de intentos serios realizados anteriormente con el objetivo de alcanzar mejoras, y a pesar de no haber cristalizado en un cambio dramático, siembran las bases para posteriores cambios que son factibles a consecuencia del aprendizaje alcanzado con las mejoras paulatinas realizadas anteriormente. $Y$ es que la reingeniería "drástica" tal como la proponen Hammer y Champy (1994) puede que no ocurra si no se dan intentos fallidos previos.

Mientras Hammer y Champy afirman que reingeniería difiere de reestructuración ( $y$ de reorganización, delaying, downsizing, y otras estrategias) en la práctica difieren en el proceso, no en los efectos, ya que uno de los lados oscuros de la aplicación de reingeniería es la pérdida de puestos de trabajo que se produce. Afirman, Hammer y Champy (1994: 56), que las compañías que emprenden la reinge. niería no sólo comprimen los procesos horizontalmente, sino también verticalmente. Los trabajadores realizan hoy aquella parte del oficio que antes ejecutaban los gerentes. En el trasfondo del planteamiento lo que ocurre es que los trabajadores se capacitan mucho más en tareas que antes compartían, lo que conlleva a un desplazamiento o reducción de empleo. Esto se traduce en una contradicción entre el anterior planteamiento y el que los autores le dan al rol que debe jugar la informática en su papel capacitador.

Hammer y Champy (1994) consideran que la forma como se ve y utiliza la informática pudiera crear una ventaja en la eliminación de los especialistas, y eso como consecuencia de la visión inductiva como se utiliza la informática en la búsqueda de soluciones a problemas que aún no existen. Ellos afirman que el error que cometen las organizaciones es ver a la tecnología a través del lente de los procesos existentes, señalando algunos 
ejemplos de organizaciones que buscando un método para procesamiento más rápido de información, encontraron soluciones que permitieron prescindir totalmente de procesos donde intervenia el hombre. Detrás de este planteamiento existe una búsqueda por la eliminación de personal dedicado a tareas muy específicas a través del uso de la automatización, y eso es parte de la reingeniería tal como la ven Hammer y Champy.

Según (Casio, 1993) un número creciente de estudios muestran que después de downsizing, la moral declina invariablemente, y los empleados que sobreviven se vuelven más desanimados, retraídos y adversos al riesgo. Los síntomas son tan comunes que a esa situación se le ha acuñado el nombre de "síndrome del sobreviviente", que es contrario a lo que ofrecen las ideas de Hammer $y$ Champy acerca de que reingenieria hace al trabajo más satisfactorio y recompensante.

Cuando se automatiza, por lo general, se piensa en la posibilidad de sustituir un proceso que está siendo ejecutado por seres humanos por otro donde la tecnología de información pasa a formar parte importante del proceso, con la esperanza de aumentar la productividad, automatizando labores manuales, consideradas lentas, poco seguras y poco precisas, por un proceso más seguro y rápido que en lo posible funcione en forma ininterrumpida, y que además se traduzca en beneficios en ahorro de personal laborando en esos procesos. Sin embargo, tal ahorro en personal y el posible ahorro en duplicación de tareas y de maquinaria, pronto se ve superado por los nuevos costos que se generan en la automatiza- ción, tales como los correspondientes al software, al hardware, $y$ al mantenimiento de los SI ya automatizados.

En el caso especifico de lo que es la reestructuración y la reingeniería, estas técnicas se orientan al desarrollo de sistemas tácticos, ya que buscan obtener mejoras cuantitativas, tales como la disminución de los costos y la disminución del recurso humano dedicado a las actividades de información, en especial las relacionadas con el control administrativo. Se busca hacer énfasis en mejorar lo que se hace, bien sea en los procesos manuales o en los automatizados. El objetivo primordial está en hacer que los actuales procesos funcionen mejor.

Aunque el automatizar no es una garantía de mejoras en la calidad del tiempo de los procesos que funcionan en la organización, ciertamente existe una ventaja generada al aplicar un nuevo diseño organizacional, bien sea vía reestructuración o reingeniería, ya que ambas focalizan en la mejora de la calidad de los procesos, en la disminución al máximo de errores, y en la disminución del tiempo de respuesta tras una solicitud. El aporte significativo de la automatización a este nivel es de tipo táctico y de corto plazo, ya que consiste en una búsqueda por incrementar la eficiencia de los procesos. Antiguos procesos que se ejecutaban manualmente 0 en forma automatizada, ahora-gracias a la aplicación de reestructuración o de reingeniería- se realizan más eficientemente. La parte negativa de este asunto es que tales SI conforman soluciones no integradas dentro de la organización, lo que conlleva a la creación de islas automatizadas de información. 
Cuando las organizaciones introducen automatización para sustituir partes del trabajo intensivo realizado por el personal, están enfocando su plan de incorporación tecnológica hacia tareas repetitivas y rutinarias, tratando de resolver problemas administrativos desde un punto de vista táctico.

Si la organización combina procesos automatizados con procesos manuales, y si uno depende de la eficiencia con que se aplica el otro, existirá una alta propensión a que se generen cuellos de botella de información, que solo serán solucionados en forma manual. Lo que en un principio significó una necesidad por automatizar, ahora requiere que se solvente a través de lo que quería ser evitado, esto es, aplicar procesos manuales. Así que la causa que generó la automatización es ahora la responsable por solventar los flujos de información que se están aglomerando. Por lo tanto, automatizar procesos en la organización no es una garantía de éxito en el procesamiento de información.

Los aportes que deberian proveer las TI son de orden estratégico y no de orden táctico. Las TI deben estar orientadas a la búsqueda de las ventajas competitivas que ellas pueden potenciar. Para buscar esas ventajas competitivas que resulten de la incorporación de $\mathrm{TI}$, se debe alinear la estrategia global de la organización, con los planes de desarrollo de los sistemas de información estratégicos. Debe asegurarse que las diferentes partes de la organización sean empujadas hacia la misma dirección que señala su estrategia. Alinear los sistemas de información con su estrategia global es consecuencia de la sinergia organizacional. Según Andreu (1991:42), es difícil obtener ventajas competitivas sostenibles si los planes de TI/SI se desarrollan de manera pasiva solamente en función de la estrategia de la empresa y para apoyar a la misma; es necesario algo más: integrar las posibilidades de los SI y de las TI con la estrategia de la empresa en el momento de formularla. $Y$ es que en ese proceso organizacional se produce un aprendizaje de la organización que es determinante para el diseño, desarrollo y posterior instrumentación de los sistemas de información. Las ventajas competitivas que se pueden alcanzar como consecuencia de la integración entre tecnologias de información y sistemas de información sólo son posibles si todos los miembros de la organización participan productivamente de ese aprendizaje.

Muchas veces se invierten enormes recursos financieros en adquisición de $\mathrm{TI}$, sin la seguridad de que ellas puedan colaborar en alcanzar las metas y objetivos de la organización. Esta divergencia pudiera ser consecuencia de los diferentes niveles, experiencias y hasta intereses que tiene la gente de sistemas de información y el resto de las personas, y pudiera mantenerse por mucho tiempo sin ser detectada. Así que cuando se establece la estrategia de la organización, las $\mathrm{Tl}$ deben ser consideradas como un factor productivo, tal como lo es el recurso financiero o el recurso humano, y no como una herramienta de producción que solo es utilizada para realizar tareas sencillas en una forma más rápida y precisa.

Según Blyth (1998:258), el problema con estos métodos es que se concentran en la creación de soluciones técnicas. Se alcanzan soluciones técnicas a través de definiciones fijas y rígidas de 
conceptos organizacionales claves. A conceptos sociales como la responsabilidad no se les puede dar una definición que se sostenga a través de todas las culturas. Consecuentemente, esos enfoques establecen la necesidad de la creación de una cultura organizacional a través de la imposición de sus propias definiciones de los conceptos sociales y por lo tanto de su propia cultura.

Hay entonces que proveer un mecanismo que permita la especificación de valores sociales, donde el análisis e interpretación de la organización esté acorde con la misión, los objetivos y las estrategias que la conducirán hacia las ventajas competitivas buscadas.

Las TI, en el largo plazo, deben ser consideradas el factor diferenciador que permita ventajas competitivas de la organización. Para ello, la compenetración entre la alta gerencia y la gente de sistemas debe estar dirigida a alcanzar el entendimiento mutuo acerca de los fines y medios que la estrategia debe seguir dentro de los parámetros establecidos en la misión de la organización.

El esfuerzo se debe orientar a realizar las cosas que permitan adaptarse con éxito al entorno, considerando como parte de el, a los competidores, a los clientes, al mercado en el horizonte de largo plazo, y muy especialmente tratar de alcanzar un aumento en la calidad de los sistemas de información, para una respuesta oportuna a las necesidades del medio.

La única solución posible para competir con ventajas en un entorno informatizado es alcanzar la integración de los sistemas de información con el resto de la organización. Y la única forma para que la tecnología pueda trabajar bajo este esquema es a través de sistemas de información que sean espejos del conocimiento humano, que reflejen la forma como la gente piensa cuando realiza un trabajo.

Los nuevos SI deberán estar dirigidos a interconectarse en forma inteligente, de forma que minimicen en lo posible ese grupo de islas automatizadas que corresponde a las organizaciones del pasado. Tales sistemas de información deben estar basados en el conocimiento que existe de la realidad; las próximas generaciones de tecnologías de información para las organizaciones serán sistemas distribuidos centrados en los seres humanos.

\section{Conclusiones}

Cualesquiera de los dos enfoques del diseño organizacional, reestructuración o reingeniería, está orientado a mejorar las actividades en relación con sus elementos internos y a mejorar su relación con los clientes, para beneficio exclusivo de la organización en el corto y mediano plazo.

Cambios dramáticos o drásticos que resulten beneficiosos para la organización ocurren si ella está preparada para tales cambios. Considerar al factor humano como parte importante de esos cambios es lo que debería caracterizar a un proceso de reingenieria.

Cambios dramáticos que se reflejen en ventajas competitivas para la organización, suelen ocurrír cuando se han venido aplicando cambios paulatinos que conducen a una adecuación del entorno a esos nuevos procesos. 
Los sistemas estratégicos se orien$\tan$ hacia el desarrollo de mejores relaciones con el entorno. Se pretende alcanzar mejoras cualitativas que generen ventajas competitivas frente a los competidores.

Para desarrollar sistemas estratégicos se deben integrar las posibilidades y capacidades de los sistemas de información y las tecnologías de información, junto a la estrategia global de la organización. En esencia se requiere de un enfoque holístico que incluya una revisión sjmultánea de la estrategia de la organización, sus operaciones y la estructura de las tecnologías de información existente o por existir.

Los sistemas de información deberán ser creados con un diseño centrado en lo humano, y no con un diseño centrado en lo tecnológico como hasta ahora ha ocurrido. Tales sistemas deben estar enmarcados en la cultura propia de cada organización.

\section{Bibliografía citada}

Andreu, Rafael; Ricart Joan y Valor Josep (1991). Estrateglas y sistemas de información. Serie McGraw-Hill de Management. Madrid, España.

Ayres, R.U. (1991) "Information, computers, computer-integrated-manufacturing and productivity". Technology and Productivity: The challenge for economic policy. 349-359, París, Francia: OCDE.

Blyth, A.J.C. (1998) "Identifying requirements for the management of medical information technology". International Journal of Technology Manage- ment. IJTM. Volume 15, Nos 3/4/5 256-269. Inderscience Enterprises Ltd. Inglaterra, Reino Unido.

Casio, W. F., "Downsizing: What Do We Know? What Have We Learned?" Academy of Management Executive, 7:1 (1993), 95-104.

Chikofsky, E.J y Cross, J HIII (1990), Reverse Engineering and Design Recovery: A Taxonomy, IEEE Software, January, 13-17.

Feller, Peter H. (1993) "Reengineering: An Engineering Problem" Special Report CMU/SEI-93-SR-5. July 3

Hammer, M., (1990) "Reengineering Work: Don't Automate, Obliterate." Harvard Business Review (July-August), 104-112

Harnmer, M., and J. Champy (1994), Reingeniería. Olvide lo que usted sabe sobre cómo debe funcionar una empresa. ICasi todo está erradol, Colombia. Editorial Norma.

Itami, Hiroyuki. (1987) Mobilizing invisible assets. Harvard University Press. Cambridge, MA, USA.

Le Quesne, P.N. (1988) "Individual and Organisational Factors and the Design of IPSEs". The Computer JOUANAL. The British Computer Society. Vol 31 No. 5 October. 391-397. Cambridge University Press. Londres, Inglaterra.

Pressman, Roger (1992) Software EngineerIng. A practitioner's approach. Third Edition. McGraw-Hill, Inc. New York.

Pressman, Roger (1998) Ingenieria de Software. Un enfoque práctico. Cuarta Edición. McGraw-Hill, Inc. New York.

Wilkinson, B. (1983) The Shopfloor Politics of New Technology, Heinemann, Londres, Inglaterra. 